\title{
Correction to: Measuring consistency of interval-valued preference relations: comments and comparison
}

\section{Fang Liu ${ }^{1}$ D $\cdot$ Mao-Jie Huang ${ }^{1} \cdot$ Cai-Xia Huang ${ }^{1} \cdot$ Witold Pedrycz $^{2}$}

Received: 27 March 2021 / Revised: 28 May 2021 / Accepted: 5 June 2021 /

Published online: 7 July 2021

(c) Springer-Verlag GmbH Germany, part of Springer Nature 2021

\section{Correction to: Operational Research https://doi.org/10.1007/s12351-020-00551-z}

In the original publication of this article, it was stated that the number of quasi interval multiplicative reciprocal matrices (IMRMs) with $n$ order is

$$
C_{n(n-1) / 2}^{1}+C_{n(n-1) / 2}^{2}+\cdots+C_{n(n-1) / 2}^{n(n-1) / 2}=2^{n(n-1) / 2}-1 .
$$

In fact, the case without a quasi-positive interval is not considered for the entries above or below the diagonal of a quasi IMRM in (1). When all quasi IMRMs are computed, the number is given as

$$
C_{n(n-1) / 2}^{0}+C_{n(n-1) / 2}^{1}+C_{n(n-1) / 2}^{2}+\cdots+C_{n(n-1) / 2}^{n(n-1) / 2}=2^{n(n-1) / 2} .
$$

For example, consider the following matrix:

$$
\bar{A}_{1}=\left[\begin{array}{c|ccc} 
& x_{1} & x_{2} & x_{3} \\
\hline x_{1} & {[1,1]} & {[2,3]} & {[1 / 2,1]} \\
x_{2} & {[1 / 3,1 / 2]} & {[1,1]} & {[1 / 6,1 / 2]} \\
x_{3} & {[1,2]} & {[2,6]} & {[1,1]}
\end{array}\right],
$$

The original article can be found online at https://doi.org/10.1007/s12351-020-00551-z.

The work was supported by the National Natural Science Foundation of China (Nos. 71571054, 71871072), the Guangxi Natural Science Foundation for Distinguished Young Scholars (No. 2016GXNSFFA380004).

Fang Liu

fang272@126.com; f_liu@gxu.edu.cn

1 School of Mathematics and Information Science, Guangxi University, Nanning 530004, Guangxi, People's Republic of China

2 Department of Electrical and Computer Engineering, University of Alberta, Edmonton, AB T6R 2V4, Canada 
Eight quasi IMRMs are determined from $\bar{A}_{1}$ by considering the locations of quasi intervals such as $\bar{A}_{1}^{\prime}$ :

$$
\bar{A}_{1}^{\prime}=\left[\begin{array}{c|ccc} 
& x_{1} & x_{2} & x_{3} \\
\hline x_{1} & {[1,1]} & {[2,3]} & {[1 / 2,1]} \\
x_{2} & {[1 / 2,1 / 3]} & {[1,1]} & {[1 / 6,1 / 2]} \\
x_{3} & {[2,1]} & {[6,2]} & {[1,1]}
\end{array}\right] .
$$

Hereafter the intervals in italic stand for the quasi ones.

Moreover, according to Theorem 1 in Liu et al. (2020), there is a permutation of objectives such that any quasi IMRM can be changed to a matrix where all quasi-positive intervals are above the diagonal and the standard ones locate below the diagonal. However, it is found that Theorem 1 in Liu et al. (2020) does not hold for $\bar{A}_{2}^{\prime}$ and $\bar{A}_{3}^{\prime}$ where

$$
\bar{A}_{2}^{\prime}=\left[\begin{array}{c|ccc} 
& x_{1} & x_{2} & x_{3} \\
\hline x_{1} & {[1,1]} & {[2,3]} & {[1,1 / 2]} \\
x_{2} & {[1 / 2,1 / 3]} & {[1,1]} & {[1 / 6,1 / 2]} \\
x_{3} & {[1,2]} & {[6,2]} & {[1,1]}
\end{array}\right],
$$

and

$$
\bar{A}_{3}^{\prime}=\left[\begin{array}{c|ccc} 
& x_{1} & x_{2} & x_{3} \\
\hline x_{1} & {[1,1]} & {[3,2]} & {[1 / 2,1]} \\
x_{2} & {[1 / 3,1 / 2]} & {[1,1]} & {[1 / 2,1 / 6]} \\
x_{3} & {[2,1]} & {[2,6]} & {[1,1]}
\end{array}\right]
$$

That is, the result of Theorem 1 in Liu et al. (2020) is wrong. An obvious reason is that the number of quasi IMRMs is bigger than that of the permutations due to the following inequality:

$$
2^{n(n-1) / 2} \geq n !, \quad n \geq 3 .
$$

A correct statement of the theorem is provided below:

Theorem 1 Assume that a quasi IMRM $\bar{A}^{\prime}=\left(\bar{a}_{i j}^{\prime}\right)_{n \times n}$ is constructed from a standard IMRM $\bar{A}=\left(\bar{a}_{i j}\right)_{n \times n} . \bar{a}_{i j}^{\circ}$ stand for the quasi-positive intervals in $\overline{A^{\prime}}$ for $i \in V_{i}$ and $j \in V_{j}$ where $V_{i, j}$ are the subsets of $\{1,2, \ldots, n\}$. If there is a permutation of objectives $\sigma$ such that

$$
\sigma(i)<\sigma(j), \quad \text { or } \quad \sigma(i)>\sigma(j),
$$

for $\forall i \in V_{i}$ and $\forall j \in V_{j}$, then $\overline{A^{\prime}}$ can be changed to the matrix where all the entries above the diagonal are quasi-positive intervals and all the entries below the diagonal are standard intervals.

Proof Suppose that there is a permutation of objectives $\sigma$ such that $\sigma(i)<\sigma(j)$ for $\forall i \in V_{i}$ and $\forall j \in V_{j}$. This means that under the permutation $\sigma$, the quasi-positive entries 
are above the diagonal. Since the number of quasi-positive intervals is $n(n-1) / 2$, the entries above the diagonal are all quasi-positive and the result is satisfied.

On the other hand, let us assume that there is a permutation of objectives $\sigma$ such that $\sigma(i)>\sigma(j)$ for $\forall i \in V_{i}$ and $\forall j \in V_{j}$. Without loss of generality, we only consider the following matrix:

$$
{\overline{A^{\prime}}}^{\prime}=\left(\bar{a}_{i j}^{\prime}\right)_{n \times n}=\left[\begin{array}{c|ccc|c|c} 
& \multicolumn{2}{c}{x_{1}} & x_{2} & \cdots & x_{n} \\
\hline x_{1} & {[1,1]} & \left.a_{12}^{-}, a_{12}^{+}\right] & \cdots & a_{1 n}^{-}, a_{1 n}^{+} \\
x_{2} & {\left[a_{21}^{+}, a_{21}^{-}\right]} & {[1,1]} & \cdots & {\left[a_{2 n}^{-}, a_{2 n}^{+}\right]} \\
\vdots & \vdots & \vdots & \ddots & \vdots \\
x_{n} & {\left[a_{n 1}^{+}, a_{n 1}^{-}\right]} & {\left[a_{n 2}^{+}, a_{n 2}^{-}\right]} & \cdots & {[1,1]}
\end{array}\right] .
$$

In order to move the quasi-positive entries below the diagonal to the positions above the diagonal, one only needs to change the permutation of objectives as $\left(x_{n}, x_{n-1}, \ldots, x_{1}\right)$. Then one can obtain the changed matrix as

$$
\bar{A}_{c}^{\prime}=\left[\begin{array}{c|ccccc} 
& x_{n} & x_{n-1} & \cdots & x_{1} \\
\hline x_{n} & {[1,1]} & {\left[a_{n(n-1)}^{+}, a_{n(n-1)}^{-}\right]} & \cdots & {\left[a_{n 1}^{+}, a_{n 1}^{-}\right]} \\
x_{n-1} & {\left[a_{(n-1) n}^{-}, a_{(n-1) n}^{+}\right]} & {[1,1]} & \cdots & a_{(n-1) 1}^{+}, a_{(n-1) 1}^{-}
\end{array}\right] .
$$

It is seen from $\bar{A}_{c}^{\prime}$ that all the entries above the diagonal are quasi-positive entries. The proof is completed.

\section{Declarations}

Conflict of interest The authors declare that they have no conflict of interest.

\section{References}

Liu F, Huang MJ, Huang CX, Pedrycz W (2020) Measuring consistency of interval-valued preference relations: comments and comparison. Oper Res Int J. https://doi.org/10.1007/s12351-020-00551-Z

Publisher's Note Springer Nature remains neutral with regard to jurisdictional claims in published maps and institutional affiliations. 